\title{
Springer
}

Manuscript for Review

\section{Saccadic tracking of targets mediated by the anterior-lateral eyes of jumping spiders}

\begin{tabular}{|r|l|}
\hline Journal: & Journal of Comparative Physiology - A \\
\hline Manuscript ID: & JCPA-Jan-12-0005.R1 \\
\hline Manuscript Type: & Original Paper \\
\hline Aute Submitted by the & 14-Mar-2012 \\
\hline Complete List of Authors: & $\begin{array}{l}\text { Zurek, Daniel; Macquarie University, Department of Biology } \\
\text { Nelson, Ximena; University of Canterbury, Department of Biological } \\
\text { Sciences }\end{array}$ \\
\hline Keywords: & Vision, Salticidae, Saccade, Motion detection, Spider \\
\hline
\end{tabular}

\section{SCHOLARONE \\ Manuscripts}


1

3

7

8

Saccadic tracking of targets mediated by the anterior-lateral eyes of jumping spiders

Daniel B. Zurek ${ }^{1 *}$ and Ximena J. Nelson ${ }^{2}$

${ }^{1}$ Department of Biological Sciences, Macquarie University, Sydney, NSW 2109 Australia

*corresponding author, e-mail: daniel.zurek@gmail.com, tel: +61 9850 1308, fax: +61 9850 9231

${ }^{2}$ School of Biological Sciences, University of Canterbury, Private Bag 4800, Christchurch, New Zealand, e-mail: ximena.nelson@canterbury.ac.nz

Keywords: Vision, Salticidae, Motion detection, Spider, Saccade 


\begin{abstract}
The modular visual system of jumping spiders (Salticidae) divides characteristics such as high spatial acuity and wide-field motion detection between different pairs of eyes. A large pair of telescope-like anterior-median (AM) eyes is supported by 2-3 pairs of 'secondary' eyes, which provide almost 360 degrees of visual coverage at lower resolution. The AM retinae are moveable and can be pointed at stimuli within their range of motion, but salticids have to turn to bring targets into this frontal zone in the first place. We describe how the frontfacing pair of secondary eyes (anterior lateral, $\mathrm{AL}$ ) mediates this through a series of wholebody 'tracking saccades' in response to computer-generated stimuli. We investigated the 'response area' of the AL eyes and show a clear correspondence between the physical margins of the retina and stimulus position at the onset of the first saccade. Saccade frequency is maximal at the margin of $A L$ and $A M$ fields of view. Furthermore, spiders markedly increase the velocity with which higher magnitude tracking saccades are carried out. This has the effect that the time during which vision is impaired due to motion blur is kept at an almost constant low level, even during saccades of large magnitude.
\end{abstract}


Introduction

Visually-guided behaviour is mirrored by eye design, a fact that is particularly well illustrated in terrestrial arthropods. The exoskeleton poses an inherent limitation to arthropod size, forcing trade-offs in the evolution of eye design. Many eye characteristics, such as temporal and spatial resolution, contrast sensitivity, and colour vision compete with each other, and only a sufficiently large eye can combine all of these abilities to a high level of performance. For example, the compound eyes of libellulid dragonflies, important aerial predators of the insect world, enable their bearers to carry out visually demanding tasks, such as identifying and chasing conspecifics, as well as resolving small, fast-moving prey while flying at high speed themselves (Labhart and Nilsson 1995). These eyes represent a pinnacle in compound eye design, but are also huge when compared to body size, and represent substantial energy investment (Laughlin et al. 1998).

Jumping spiders (Salticidae) exhibit unusually complex, visually mediated behaviour (Nelson and Jackson 2011a;b). The spider's cephalothorax carries an array of three to four pairs of eyes, each of which adds specific capabilities to the system, such as wide field of view, high spatial acuity, motion sensitivity, and colour vision (Homann 1928, Land 1971 Williams and McIntyre 1980, Yamashita and Barth 1985). Due to their small size, it is not possible for salticids to realise all of these traits in a single pair. Instead they have evolved an ingenious way of dividing the various zones of larger, more versatile eyes into multiple, more specialised eyes (Harland et al. 2012). Together, they form an intricate modular visual system that achieves higher spatial resolution and wider field of view than any insect, with simple eyes that are small compared to compound eyes. However, this functional division is perhaps not as strict as suggested by much of the literature and raises interesting questions about the integration of the input from the different eye pairs (Forster 1979; Zurek et al. 2010). A similar type of functional division has been described in a larger hunting spider, the ctenid Cupiennius salei. In C. salei, the fields of view of the forward-facing posteriormedian (PM)and anterior-median (AM) eyes overlap; the PM pair supports target detection, and the AM target discrimination (Schmid 1998). Movement detected by the PM eyes leads to heightened activity of the AM eye muscles, which can however also be elicited by flickering, non-moving stimuli (Fenk and Schmid 2011). 
The beautiful optics of the large forward-facing principal, or anterior median (AM), eyes of salticids has been extensively investigated (Eakin and Brandenburger 1971; Blest et al. 1990; Land 1969a; Williams and McIntyre 1980), as has their role in visually-guided behaviour (Harland and Jackson 2000). At the expense of a large field of view, this pair of eyes features spatial acuity (form vision) unparalleled among terrestrial invertebrates, and supports colour vision (Williams and McIntyre 1980). Another noteworthy characteristic of the AM eyes is that while the horizontal field of view of each retina is less than five degrees, these eyes compensate by performing complex movement routines to explore a greater field of view, extending ca. $28^{\circ}$ to either side of the body axis (Land 1969b, Harland and Jackson 2004). Most of the research on jumping spider vision has focused on the AM eyes, while the role of the 'secondary' pairs of eyes (anterior lateral, AL, posterior lateral, PL and the posterior median, or PM, eyes (vestigial in modern Salticoida, (Maddison and Hedin 2003)) is usually assumed to be limited to motion detection. However, the AL eyes seem to play a pivotal role in regulating behaviour and have characteristics which suggest that their classification as pure motion detectors may be somewhat simplistic: they not only possess a wide field of view in which small target motion is reliably detected, but also have remarkable acuity, as defined histologically (Eakin and Brandenburger 1971), optically (Land, 1985), and behaviourally (Zurek et al. 2010).

The AL eyes mediate orientation turns in response to visual stimuli in their field of view (Land 1971; Duelli 1978; Komiya et al. 1988; Zurek et al. 2010), and as such they coordinate visual input with body movement to bring the target into a frontal field of view. Like saccadic eye- or head-movements in vertebrates and invertebrates, these whole-body optomotor responses have the goal of bringing part of the visual field into a 'fixation region' (Rossel 1980), which in the case of salticids means that the target can be investigated with the high acuity, colour sensitive, AM eyes. There is evidence that the AL eyes also play a role in quickly categorising moving objects and in the initiation of appropriate responses, such as chasing and prey capture (Forster 1979; Zurek et al. 2010). In this function, they are likely to mediate not only detection, but also tracking of movement in the frontal visual field. By 'deciding' what to respond to, and preferentially eliciting orientation turns to a subset of stimuli for accurate classification by the AM eyes and subsequent behavioural response, the AL eyes might almost be classified as the first level decision-makers of salticid visual 


\section{1}

behaviour. Here, we investigate how the design of the AL eyes of a bark-dwelling salticid, Servaea vestita, correlates with the first levels of visual behaviour: visual orienting and visual tracking. This is part of ongoing research with the aim to redress the balance of knowledge about the respective roles of primary and secondary eyes, while placing this in a behavioural and ecological context.

\section{Methods}

Spider rearing methods, lab conditions and basic experimental procedures were as described in Zurek et al. (2010), so the experimental protocol will be described here in abbreviated form. Spiders were restrained by placing them on a large diameter plunger with a foam base. A plastic vial with holes was placed over the plunger and lightly pressed down on the spider such that the cephalothorax of the restrained animal protruded through one of the holes. This allowed us to position the spider while restraining it without the potential adverse effects of anaesthesia. All eyes except for the AL eyes were then covered with an opaque, non-toxic and removable dental silicone (Coltene Whaledent President light body polyvinyl siloxane) following methods described by Zurek et al. (2010). A 3x3 mm cork cube connected to a thin wire was then attached to the cephalothorax with a drop of bee's wax. This makes it possible to suspend the spider from a crocodile clip on a flexible arm, which can be precisely positioned in relation to the stimulus presentation screens. Spiders were starved for 7 days before testing.

For stimulus presentation, we used two 17" TFT screens (Samsung 743B, resolution $1280 \times 1024$ px, $75 \mathrm{~Hz}$, response time 5ms; Samsung Electronics, Seoul, South Korea) that were positioned at an angle of 120 degrees with respect to each other, placed on a vibration isolation table (Kinetic Systems, Boston, MA, USA). Colour and brightness levels of the screens were measured and calibrated using an Eye One Display V.2 colorimeter (X-Rite Incorporated. Grand Rapids, MI, USA) controlled via the software ColorHCFR v.2.1 (HCFR Colorimeter team, Paris, France) on an external PC. Stimuli were generated using VPixx V2.36 (VPixx Technologies Inc., Montreal, QC, Canada) on a Mac Pro (2x 2.8 GHz Quad Core Xeon, 4 GB RAM) and a Macbook Pro (2.4 GHz Core 2 Duo, 4 GB RAM) (Apple Computer Inc., 
Cupertino, CA, USA). Stimulus contrast was defined as the difference between the luminance of the stimulus and the background, divided by the luminance of the background (Weber contrast). The stimuli were always darker than the background, as relevant objects moving into the field of view of the AL eyes are usually darker than the background (Duelli, 1978). For a spider $150 \mathrm{~mm}$ away from the screen, 1 pixel was 0.1 degrees wide. Suspended spiders were positioned $150 \mathrm{~mm}$ from the centre of each of two screens, facing their intersection. In this position, the screens filled the complete field of vision of the AL eyes (Fig. 1). This stimulus presentation method has proven to be highly effective at eliciting orienting responses from jumping spiders (Zurek et al., 2010) and is more effective than presenting stimuli on a rotating drum (e.g., Land, 1971).

Suspended spiders held on to a $160 \mathrm{mg}$ polystyrene ball (diameter $15 \mathrm{~mm}$ ) marked with crosshair lines. While the ball was heavier than the average weight of an adult spider (mean $\pm S D$; adult female $64.1 \mathrm{mg} \pm 16.6 \mathrm{mg}, \mathrm{N}=45$; adult male $56.8 \mathrm{mg} \pm 23.4 \mathrm{mg}, \mathrm{N}=26$ ), spiders could easily turn the ball and hold on to it for several hours without becoming fatigued (Zurek et al.2010). However, there was the potential that the angles turned by the spiders and the durations of turns could be affected by the weight of the ball. For the following reasons we are confident that this is not the case: the moment of inertia that the spider has to overcome in order to turn can be assumed to lie between those of a rectangle and a sphere with the same mass as the spider (Land 1972). For a sphere, the moment of inertia is given as $I_{\text {sphere }}=2 / 5 * m_{\text {spider }} * r^{2}$ and for a rectangle $I_{\text {rectangle }}=1 / 12 * m_{\text {spider }} *\left(a^{2}+b^{2}\right)$. Accordingly, for adult female S. vestita, this moment of inertia should lie between $409 \mathrm{mg}$ $\mathrm{mm}^{2}$ and $389 \mathrm{mg} \mathrm{mm}^{2}$; for convenience we assume it to be $400 \mathrm{mg} \mathrm{mm}^{2}$. The moment of inertia for turning the polystyrene ball is $3600 \mathrm{mg} \mathrm{mm}^{2}$. While this is nine times greater than that of the spider itself, Land (1972) has shown that even objects with moments of inertia 400 times greater than that of a spider have no effect on the accuracy of turning or the pattern of leg movement, and only a tiny effect on turn velocity.

In this study, we use the term 'saccade' to describe a single, discrete turn, and the term 'orientation response' for all turning movements, expressed either as a single saccade or a series of saccades, in response to moving visual stimuli. When the spider's body is held in place, saccades lead to an obvious spin of the ball in the direction opposing that of the 


\section{1}

stimulus. Saccades very rarely occur spontaneously and, due to their high speed and discrete nature, they are easily distinguished from walking (Zurek et al. 2010).

\section{Response area tests}

We used dark dot stimuli on a brighter background (grey with faint gridlines) to elicit orientation responses from suspended spiders. In all trials we presented horizontally moving dot stimuli with characteristics known to elicit maximal response rates ( $4^{\circ}$ diameter with maximum contrast, moving at $9^{\circ} / \mathrm{s}$ (Zurek et al. 2010)). Single dots were generated the posterior periphery of the screens, moving towards the centre. The dots were presented to 30 adult females at vertical elevations ( $y$-level) between $-40^{\circ}$ and $+40^{\circ}$, once from the left and once from the right side at each y-level, in random order. Stimuli higher or lower than $\pm 40^{\circ}$ never elicited orientation responses.

After allowing the spider to acclimate to the experimental set-up for at least $5 \mathrm{~min}$, stimuli were presented every $2 \mathrm{~min}$. Trials were filmed at $30 \mathrm{fps}$ with a Sony digital video camera (Sony DCR HC52E, Sony Corporation, Tokyo, Japan), positioned $30 \mathrm{~cm}$ behind the spider. We calculated the percentage of stimuli that elicited orientation responses at every $y$-level and calculated the means and standard deviations of the horizontal positions where initial orientation responses occurred. The position of the leading edge of the stimulus at the onset of the first saccade was used for graphing results. We refer to the area formed by a line through these points as the eyes' 'response area'.

\section{Saccade tests}

Six female spiders that had shown high activity levels in preliminary trials were selected for testing. Spiders were placed as described for the response area tests. Stimuli were presented at horizon level on the left or right screen in random order $(N=100)$. To prevent habituation there was a 2 min rest between trials and a 15 min pause after 20 trials. Spiders reacted to moving dots with a series of saccades. A video camera was positioned $20 \mathrm{~cm}$ above the spider, and a mirror angled at $45^{\circ}$ was placed below the spider. This configuration simultaneously allowed us to record the stimulus position when a saccade occurred, the angle turned by the spider (saccade magnitude), and the time it took to complete each saccade (saccade duration). These parameters enabled us to calculate saccade velocity. 


\begin{abstract}
Video footage was scored using QuickTime Player 7.6.6 (Apple Computer Inc., Cupertino, CA, USA) and measurements of angles turned were determined using Screen Protractor 3.2 (Iconico Inc., New York, NY, USA).
\end{abstract}

\title{
Results
}

Saccades occurred shortly after stimuli entered the visual field of the AL eyes at all tested levels on the y-axis (Fig. 1). The average leading edge positions of a stimulus at the onset of the first saccade were within the opththalmoscopically determined AL eye field of view of Plexippus sp. (former name of Servaea) by Land (1985), and corresponded closely with the margins of a more detailed map by O'Carroll (1989), which is shown in Fig.1. Saccades commenced when the $4^{\circ}$ dot was completely within the field of view, indicating that perception of the trailing edge is necessary for a response.

The likelihood that a spider turned in response to a stimulus was close to $100 \%$ in large parts of the visual field (Fig. 1). At y-levels from $-30^{\circ}$ to $+12^{\circ}$ ), orientation responses were elicited in over $90 \%$ of trials, with stimuli entering the visual field at $-10^{\circ}$ eliciting turns in all trials. The widest visual angle (on average $56.14^{\circ} \pm 9.83^{\circ}, \mathrm{N}=30, \mathrm{n}=52$ ) at which orientation responses were observed was at vertical level of $-27.5^{\circ}$.

Orientation responses consisted of a series of individual saccades, with varying frequencies, magnitudes, and durations. When the stimulus crossed the margin of the visual field and travelled through it to the central sagittal plane, saccade frequency increased as the stimulus travelled through zones of increasing spatial acuity. Before reaching the frontal areas of highest receptor density and binocular overlap saccade frequency dropped (Fig. 2). We observed a maximum saccade frequency of $0.319 \pm 0.05$ saccades per degree of stimulus movement (or 3 saccades/s at the presented stimulus velocity of $9^{\circ} / \mathrm{s}$ ) at stimulus positions between $25^{\circ}$ and $30^{\circ}$ laterally.

There was a linear relationship between average saccade magnitude and stimulus position, but where 'perfect' tracking of the stimulus would be represented by a slope of 1 , in practice saccade magnitude corresponded closely to stimulus position but started to undershoot it at stimulus angles greater than $20^{\circ}$ (Table 1, Fig. 2) We obtained a small 
number of orienting responses outside of $60^{\circ}$ laterally, which is where the retina ends according to opthalmoscope data (O'Carroll 1989). These responses were too frequent to be random events (Zurek et al. 2010) and the angles turned were consistent with stimulus position. These rare occurrences events might have been caused by a combination of individual differences in eye size and/or slight misalignments when positioning spiders in front of the testing screens.

All measured saccade characteristics (magnitude, velocity and duration) assumed greater values when the stimulus was at a greater angular position (Table 1, Fig. 3 a-c). For easier comparison, saccade magnitude and velocity data were normalised to percentages of their respective highest value. This revealed a strong positive correlation between the two characteristics (Table 1, Fig. 4a). In other words, larger saccades were carried out at higher speed, which had the effect of maintaining saccade duration constant even for turns of large magnitude (Fig. 4b). Nevertheless, the increase in saccade velocity was not quite enough to completely negate an increase in duration, as we observed an increase in duration with increasing saccade magnitude (Fig. 3 c,d).

Table 1 Summary of relations between different saccade characteristics (linear regressions, slope \pm SD). *Normalised data (see text for details).

\begin{tabular}{|l|l|l|l|l|}
\hline$f(x)$ & $x$ & Linear regression, slope $\pm S D$ & $R^{2}$ & Figure \\
\hline Saccade magnitude & Stimulus position & $0.6732 \pm 0.01464$ & 0.4952 & $3 \mathrm{~A}$ \\
\hline Saccade velocity & Stimulus position & $3.558 \mathrm{~s}-1 \pm 0.1146$ & 0.3089 & $3 \mathrm{~B}$ \\
\hline Saccade duration & Stimulus position & $0.9007 \pm 0.05872$ & 0.09839 & $3 \mathrm{C}$ \\
\hline Saccade magnitude* & Stimulus position & $0.8115 \pm 0.01762$ & 0.4959 & $3 \mathrm{D}$ \\
\hline Saccade velocity* & Stimulus position & $0.5653 \pm 0.01816$ & 0.3099 & $3 \mathrm{D}$ \\
\hline Saccade duration* & Stimulus position & $0.1687 \pm 0.01100$ & 0.09840 & $3 \mathrm{D}$ \\
\hline Saccade velocity* & Saccade magnitude* & $0.7525 \pm 0.009875$ & 0.7292 & 4A \\
\hline Saccade duration* & Saccade magnitude* & $0.1464 \pm 0.009543$ & 0.09832 & 4B \\
\hline
\end{tabular}




\section{Discussion}

Here, we show that the AL eyes of salticids mediate series of whole-body tracking saccades, which can be elicited by moving objects as soon as they enter the AL field of view. The frequency of these saccades is highest at the margin of the movement range of the AM eyes, where only small turns are necessary to centre the target. The increased velocity of saccades towards targets that are further away from the body axis leads to a relatively constant duration of all saccades.

The frontal visual field of salticids is formed by the overlapping fields of view of the AL and the AM eyes. While that of the AL eyes is wide and fixed, the AM eyes resemble a small high resolution telescope that can be moved, by muscles attached to the eye tubes, within some of the field of view of the AL eyes to smoothly track objects of interest (Land 1969b). In their normal position, the retinae of the AM eyes are directed forward, and their fields of view overlap with the acute zone of the AL eyes. Detection of movement by the AL eyes likely leads to movement of the AM retinae, in a similar fashion as has been described in the ctenid spider Cupiennius salei (Neuhofer et al. 2009, Fenk and Schmid 2010). The movement range of the $A M$ eye tubes is not great enough to reach every part of the AL field of view; in Phanias harfordii (formerly Metaphidippus harfordii) Land (1969a) determined that the eye tubes could move $\pm 28^{\circ}$ on a horizontal plane. Because of this limit, we would expect a process by which stimuli are 'handed over' from the AL to the AM eye. In S. vestita, we determined that the maximum saccade frequency in response to moving dots is elicited at stimulus positions between $25^{\circ}$ and $30^{\circ}$. In this 'sweet spot', small saccades were carried out in rapid succession. More peripherally, we found that less frequent saccades with higher magnitude were common. At stimulus angles below $25^{\circ}$ saccade frequency dropped dramatically, most likely because at this point the stimulus is both within the movement range of the AM eyes, as well as in the AL acute zone, and a whole-body turn is not necessary. Orientation responses mediated by the secondary eyes are carried out in an open-loop manner, meaning that visual feedback has no effect on the accuracy of the turn, which by extension implies that the eyes are essentially blind during a turn (Land 1971). Efficient tracking of moving objects requires a high sampling rate; in the case of salticids this means a high saccade frequency. Theoretically, saccade frequency can 
be maximised when the stimulus is at an angular position close to the acute zone, and this is exactly what is achieved here.

Stimuli were consistently tracked once they were within about $60^{\circ}$ from the centre of the visual field, but we found that saccades generally did not 'perfectly' track the target, but rather fell short by ca. $22 \%$. This is remarkably similar to the $26 \%$ undershoot found for saccadic head movements in response to moving stimuli in mantids (Lea and Mueller 1977), which, unlike spiders, have compound eyes. Analogous to mantid compound eyes, the combined visual field of the forward-facing simple eyes of salticids has a relatively large acute zone, which might explain this "fixation deficit" (Mittelstaedt 1957): if the frontal acute zone of the eye is large enough, saccade magnitudes smaller than the lateral angular position of the stimulus are sufficient to bring it into a region of high acuity.

The velocity and duration characteristics of salticid whole-body saccades accentuate their apparent similarity to vertebrate eye saccades. As found in the saccadic 'main sequence' of human eyes (Bahill et al. 1975), we observed a marked increase in saccade velocity at larger saccade magnitudes in the whole-body saccades of salticids. This, in fact, is a defining feature of saccadic movements in general, and comes about at least partly due to inertia: at small saccade magnitudes, the proportion of time spent accelerating and decelerating the eye (or head) is larger than the proportion spent in mid-saccade, which is one reason why large magnitude saccades are carried out proportionally faster. For salticids specifically, this has the beneficial effect of keeping the time spent turning relatively constant, which may serve an important function in the open-loop fixation system described by Land (1971). When the time spent in mid-turn, during which vision is impaired by motion blur, is constant, extrapolating the future angular position of a moving stimulus becomes more reliable.

Recently, Aptekar et al. (2012) showed that Drosophila melanogaster utilises two distinct motion tracking subsystems, one of which is suggested to engage body saccades towards peripheral targets, after which the other subsystem can smoothly track the now centred stimulus. These systems are reminiscent of the behaviour mediated by each of the two forward-facing eye pairs of salticids. Strategies that combine saccadic acquisition of peripheral sources of movement with smooth frontal fixation appear to be realised in insects, spiders, and primates; animals with fundamentally different visual systems. While 
this does not necessarily mean that we will find the same corollaries, the significance of these convergent solutions should not be ignored.

\section{Acknowledgements}

We thank María Castillo-Pando (animal care), Rowan McGinley (spider collection), Ben Fanson and Eirik Søvik (helpful discussions). This work was supported by an Australian Research Council Discovery Grant (DP0772517) to XJN and by a Macquarie University Research Excellence Scholarship (DBZ).

\section{References}

Aptekar, JW, Shoemaker, PA, and Frye, MA (2012). Figure tracking by flies Is supported by parallel visual streams. Curr Biol, 22, 1-6

Bahill, A, Clark, M, and Stark, L (1975). The main sequence, a tool for studying human eye movements. Mat Biosci, 24, 191-204.

Blest, AD, O'Carroll, DC, and Carter, M (1990). Comparative ultrastructure of Layer I receptor mosaics in principal eyes of jumping spiders: the evolution of regular arrays of light guides.

Cell Tis Res, 262, 445-460.

Duelli, $\mathrm{P}$ (1978). Movement detection in the posterolateral eyes of jumping spiders (Evarcha arcuata, Salticidae). J Comp Physiol A, 124, 15-26.

Eakin, R and Brandenburger, J (1971). Fine Structure of Eyes of Jumping Spiders. J Ultrastruct Res, 37, 618-663.

Fenk, LM and Schmid, A (2010). The orientation-dependent visual spatial cut-off frequency in a spider. $J$ Exp Biol, 213, 3111-3117.

Fenk, LM and Schmid, A (2011). Flicker-induced eye movements and the behavioural temporal cut-off frequency in a nocturnal spider. J Exp Biol, 214, 3658-3663.

Forster, L (1979). Visual mechanisms of hunting behaviour in Trite planiceps, a jumping spider (Aranae: Salticidae). NZ J Zool, 6, 79-93.

Harland, D and Jackson, RR (2000). "Eight-legged cats" and how they see-a review of recent research on jumping spiders (Araneae: Salticidae). Cimbebasia, 16, 231-240.

Harland, D and Jackson, RR (2004). Portia Perceptions: The umwelt of an araneophagic jumping spider. In, Prete, FR (ed), Complex Worlds from Simpler Nervous Systems. MIT Press, Cambridge, pp. 5-40. 
Harland, D, Li, D, and Jackson, RR (2012). How jumping spiders see the world. In, Lazareva, OF, Shimizu, T, and Wasserman, EA (eds), How Animals See the World: Comparative Behavior, Biology, and Evolution of Vision. Oxford University Press, New York, pp. 133-164.

Komiya, M, Yamashita, $\mathrm{S}$, and Tateda, $\mathrm{H}$ (1988). Turning reactions to real and apparent motion stimuli in the posterolateral eyes of jumping spiders. J Comp Physiol A, 163, 585592.

Labhart, T and Nilsson, D (1995). The dorsal eye of the dragonfly Sympetrum: Specializations for prey detection against the blue sky. J Comp Physiol A, 176, 437-453.

Land, MF (1969a). Structure of the retinae of the principal eyes of jumping spiders (Salticidae: Dendryphantinae) in relation to visual optics. J Exp Biol, 51, 443-470.

Land, MF (1969b). Movements of the retinae of jumping spiders (Salticidae:

Dendryphantinae) in response to visual stimuli. J Exp Biol, 51, 471-493.

Land, MF (1971). Orientation by jumping spiders in the absence of visual feedback. J Exp Biol, 54, 119-139.

Land, MF (1972). Stepping movements made by jumping spiders during turns mediated by the lateral eyes. J Exp Biol, 57, 15-40.

Laughlin, SB, de Ruyter van Steveninck, RR, and Anderson, JC (1998). The metabolic cost of neural information. Nat Neurosci, 1, 36-41.

Lea, J and Mueller, C (1977). Saccadic head movements in mantids. J Comp Physiol A.

Maddison, WP and Hedin, MC (2003). Jumping spider phylogeny (Araneae : Salticidae). Invertebr Syst, 17, 529.

Mittelstaedt, H (1957). Prey capture in mantids. In, Scheer, BT (ed), Recent advances in invertebrate physiology. University of Oregon, pp. 51-71.

Nelson, XJ and Jackson, RR (2011a). Anti-Predator Behaviour. In, Herberstein, ME (ed), Spider Behaviour. Cambridge University Press, Cambridge, pp. 51-73.

Nelson, XJ and Jackson, RR (2011b). Foraging Behaviour. In, Herberstein, ME (ed), Spider Behaviour. Cambridge University Press, Cambridge, pp. 5-30.

\section{Neuhofer, D, Machan, R, and Schmid, A (2009). Visual perception of motion in a hunting spider. J Exp Biol, 212, 2819-2823.}

O'Carroll, DC (1989). An optical assessment of visual performance in the eyes of hunting spiders (Araneae: Labidognatha) Wells, R (ed), The Flinders University of South Australia, Adelaide.

Rossel, S (1980). Foveal fixation and tracking in the praying mantis. J Comp Physiol A, 139, 307-331. 
Schmid, A (1998). Different functions of different eye types in the spider Cupiennius salei. J Exp Biol, 201, 221.

Williams, D and Mclntyre, $P(1980)$. The principal eyes of a jumping spider have a telephoto component. Nature, 288, 578-580.

Yamashita, S and Barth, FG (1985). Photoreceptor cells in the spider eye: Spectral sensitivity and efferent control. In, Barth, FG (ed), Neurobiology of Arachnids. Springer, New York / Heidelberg, pp. 103-117.

Zurek, D, Taylor, AJ, Evans, CS, and Nelson, XJ (2010). The role of the anterior lateral eyes in the vision-based behaviour of jumping spiders. J Exp Biol, 213, 2372-2378.

Fig. 1 Equirectangular projection of the $A L$ response area, $0 x / 0 y$ position refers to the body axis. Dots: positions of the leading edge of the stimulus (mean \pm SD) that elicited the first saccade. Continuous solid line: likelihood of responses elicited by stimuli at the respective $y$ level. Dashed line: margins of AL field of view (O'Carroll 1989)

Fig. 2 Characteristics of saccades elicited by stimuli at 0 degrees vertically (horizon). Squares, left y-axis: saccade frequency (number of saccades during $1^{\circ}$ of stimulus travel) plotted as function of stimulus position. Dots, right $y$-axis: saccade magnitude plotted as function of stimulus position. All data: mean \pm SD. Dashed line represents a theoretical spider perfectly tracking the stimulus

Fig. 3 Mean saccade magnitude (a), velocity (b) and duration (c), plotted as functions of stimulus position. (d) Comparison of saccade characteristics with normalised data. Dots: saccade magnitude. Triangles: saccade velocity. Squares: saccade duration. Linear regressions and $95 \% \mathrm{Cl}$ based on raw data, means (shown as points) plotted for clarity

Fig. 4 Saccade characteristics normalised as percentages of their respective maximum values. Means \pm SD of saccade velocity (a) and duration (b) plotted as function of saccade magnitude. Linear regressions and $95 \% \mathrm{Cl}$ based on raw data 


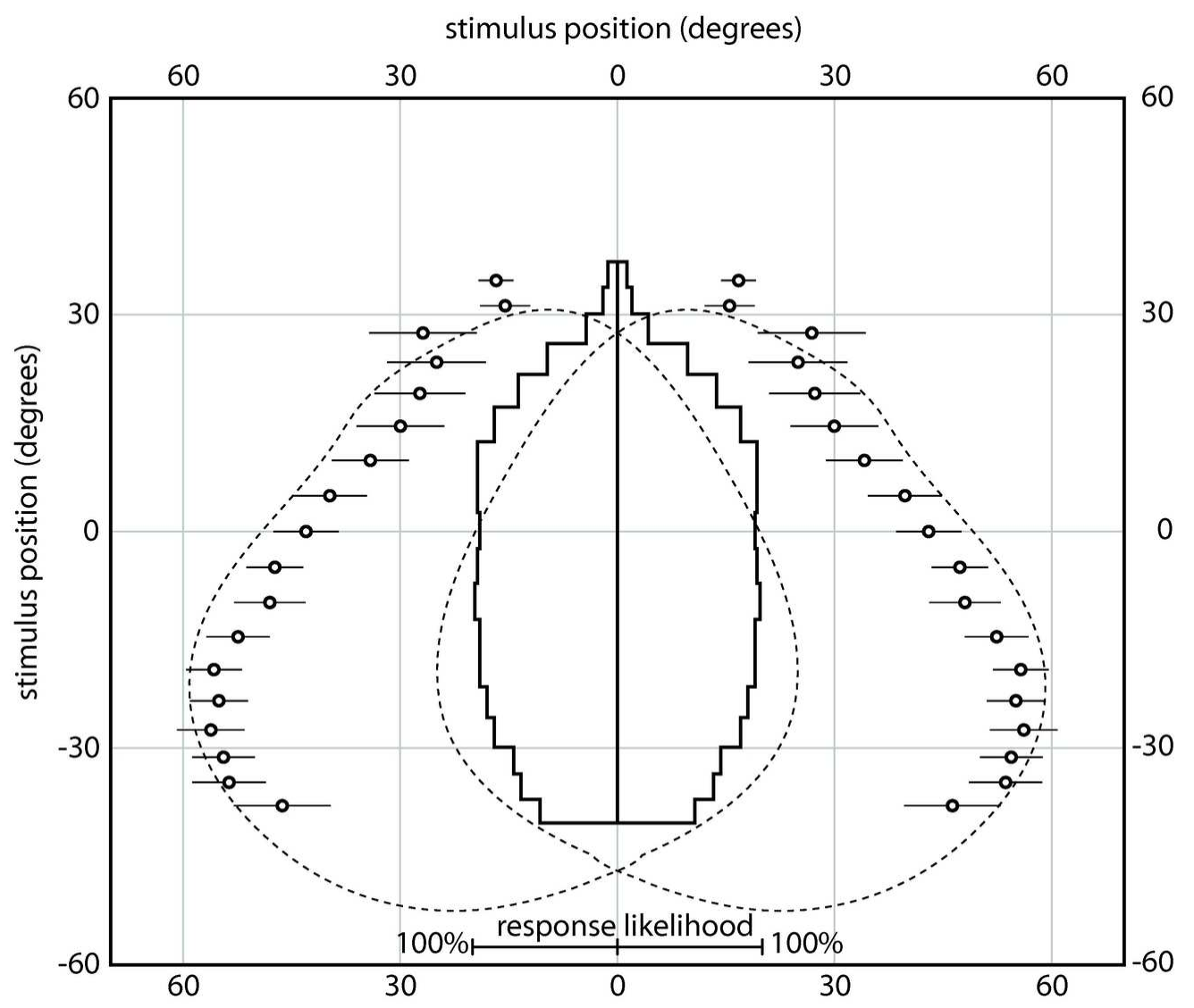

Fig. 1 Equirectangular projection of the AL response area, 0x/0y position refers to the body axis. Dots: positions of the leading edge of the stimulus (mean \pm SD) that elicited the first saccade. Continuous solid line: likelihood of responses elicited by stimuli at the respective y-level. Dashed line: margins of AL field of view (O'Carroll 1989) 


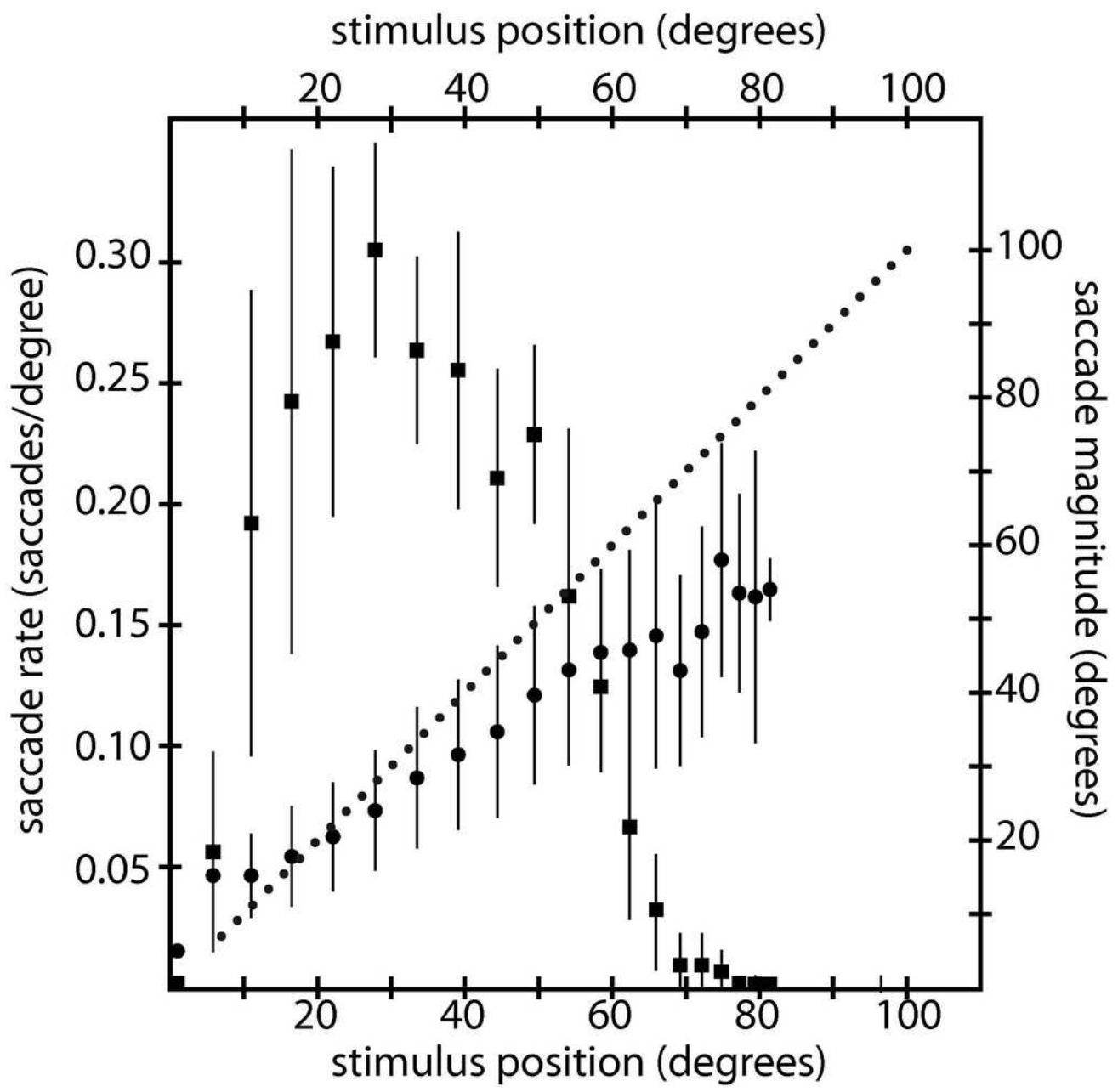

Fig. 2 Characteristics of saccades elicited by stimuli at 0 degrees vertically (horizon). Squares, left $y$-axis: saccade frequency (number of saccades during $1^{\circ}$ of stimulus travel) plotted as function of stimulus position. Dots, right $y$-axis: saccade magnitude plotted as function of stimulus position. All data: mean \pm SD. Dashed line represents a theoretical spider perfectly tracking the stimulus $86 \times 83 \mathrm{~mm}(300 \times 300 \mathrm{DPI})$ 

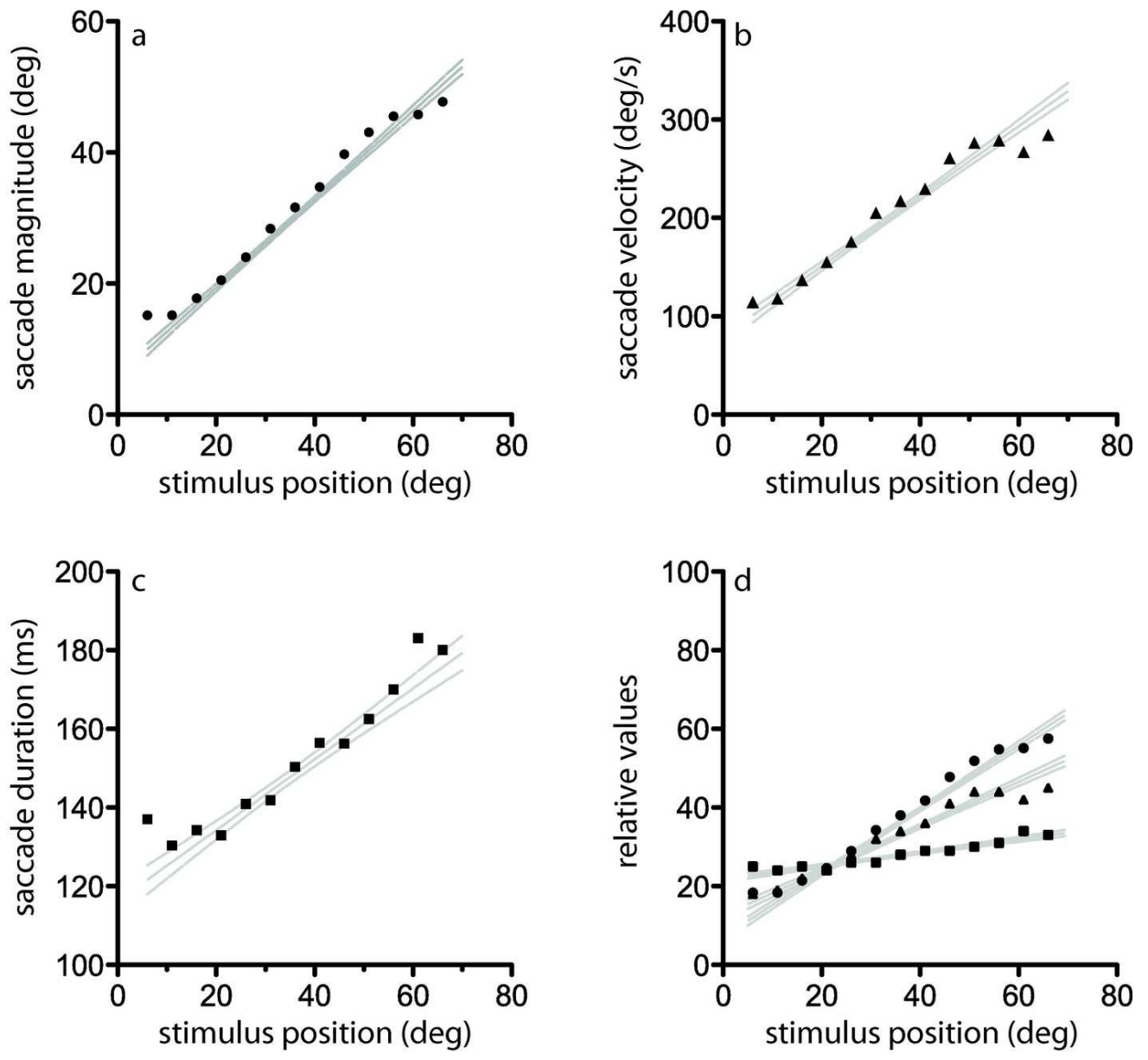

Fig. 3 Mean saccade magnitude (a), velocity (b) and duration (c), plotted as functions of stimulus position. (d) Comparison of saccade characteristics with normalised data. Dots: saccade magnitude. Triangles: saccade velocity. Squares: saccade duration. Linear regressions and $95 \%$ CI based on raw data, means (shown as points) plotted for clarity $119 \times 110 \mathrm{~mm}(300 \times 300 \mathrm{DPI})$ 

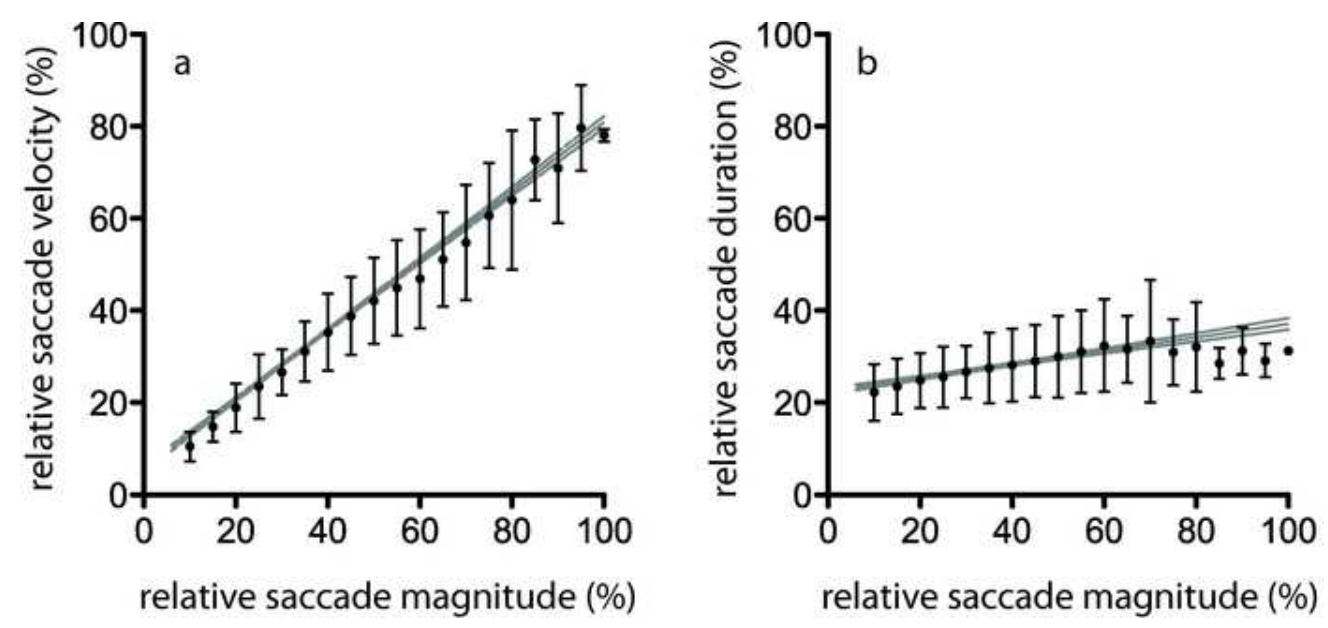

Fig. 4 Saccade characteristics normalised as percentages of their respective maximum values. Means \pm SD of saccade velocity (a) and duration (b) plotted as function of saccade magnitude. Linear regressions and $95 \% \mathrm{CI}$ based on raw data $58 \times 26 \mathrm{~mm}(300 \times 300 \mathrm{DPI})$ 